\title{
Editorial
}

\section{Applications of Fixed Point and Approximate Algorithms}

\author{
Yonghong Yao, ${ }^{1}$ Rudong Chen, ${ }^{1}$ \\ Giuseppe Marino, ${ }^{2}$ and Yeong-Cheng Liou ${ }^{3}$ \\ ${ }^{1}$ Department of Mathematics, Tianjin Polytechnic University, Tianjin 300387, China \\ ${ }^{2}$ Dipartimento di Matematica, Universitá della Calabria, 87036 Arcavacata di Rende, Italy \\ ${ }^{3}$ Department of Information Management, Cheng Shiu University, Kaohsiung 833, Taiwan \\ Correspondence should be addressed to Yonghong Yao, yaoyonghong@yahoo.cn
}

Received 8 November 2012; Accepted 8 November 2012

Copyright (C) 2012 Yonghong Yao et al. This is an open access article distributed under the Creative Commons Attribution License, which permits unrestricted use, distribution, and reproduction in any medium, provided the original work is properly cited.

As very powerful and important tools in the study of nonlinear sciences, fixed point methods have attracted so much attention. Over the last decades, fixed point techniques have been applied extensively in such diverse fields as biology, chemistry, economics, engineering, game theory, physics, and so on. The thorough study of the fixed point theory and its approach methods contained in the literature will help us to find new fixed point techniques for solving the practical problems.

The aim of this special issue is to present and extend the applications of the relatively new approaches and theories for the fixed point problems arising in mathematics and applied sciences.

This special issue includes 105 high-quality peer-reviewed papers that deal with different aspects of applications of fixed point and approximate algorithms. These papers contain some new, novel, and innovative techniques and ideas. We hope that all the papers published in this special issue can motivate and foster further scientific works and development of the research in the area of the applications of fixed point and approximate algorithms.

\section{Acknowledgments}

As Guest Editors for this special issue, we wish to thank all those who submitted papers for publication and many specialists who served as the reviewers. We highly appreciate the support from the editorial members of the journal, as well as the editorial staff of Hindawi 
Publishing Corporation. Y. Yao was supported in part by NSFC 11071279 and NSFC 71161001G0105. R. Chen was supported in part by NSFC 11071279. Y.-C. Liou was partially supported by NSC 101-2628-E-230-001-MY3.

Yonghong Yao

Rudong Chen

Giuseppe Marino

Yeong-Cheng Liou 


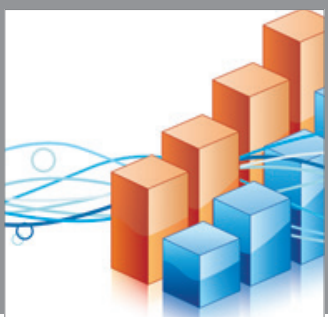

Advances in

Operations Research

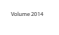

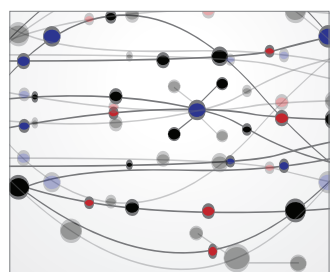

\section{The Scientific} World Journal
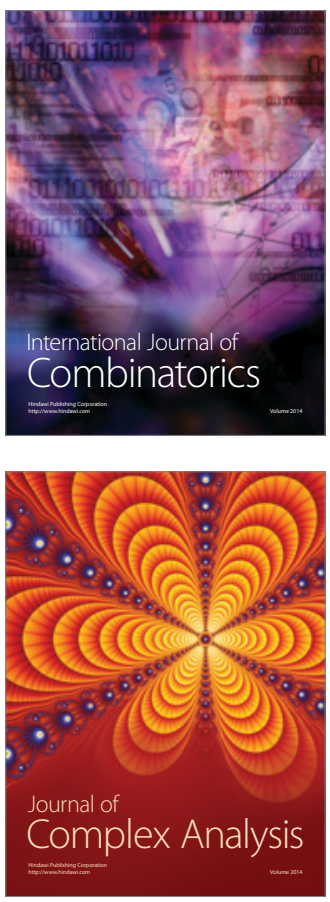

International Journal of

Mathematics and

Mathematical

Sciences
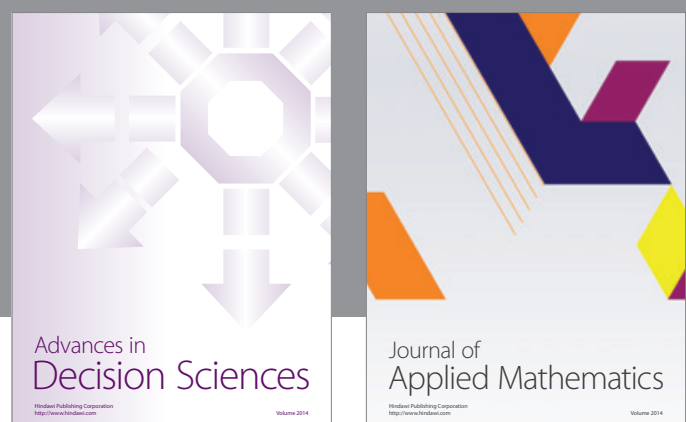

Journal of

Applied Mathematics
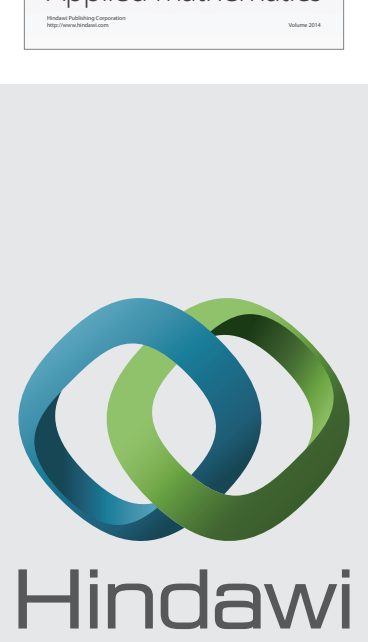

Submit your manuscripts at http://www.hindawi.com
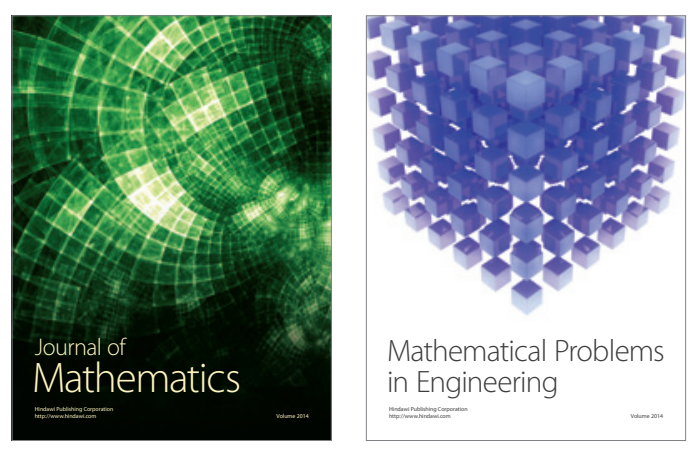

Mathematical Problems in Engineering
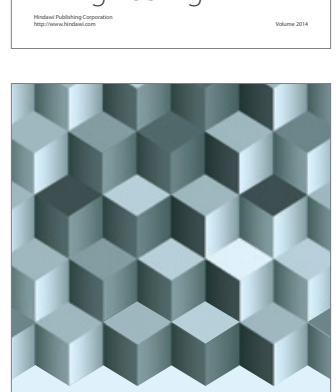

Journal of

Function Spaces
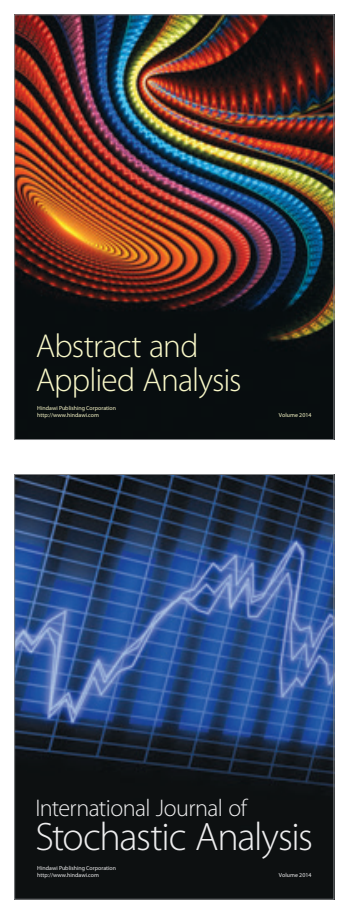

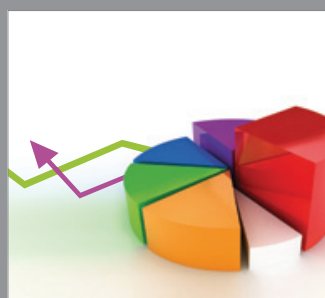

ournal of

Probability and Statistics

Promensencen
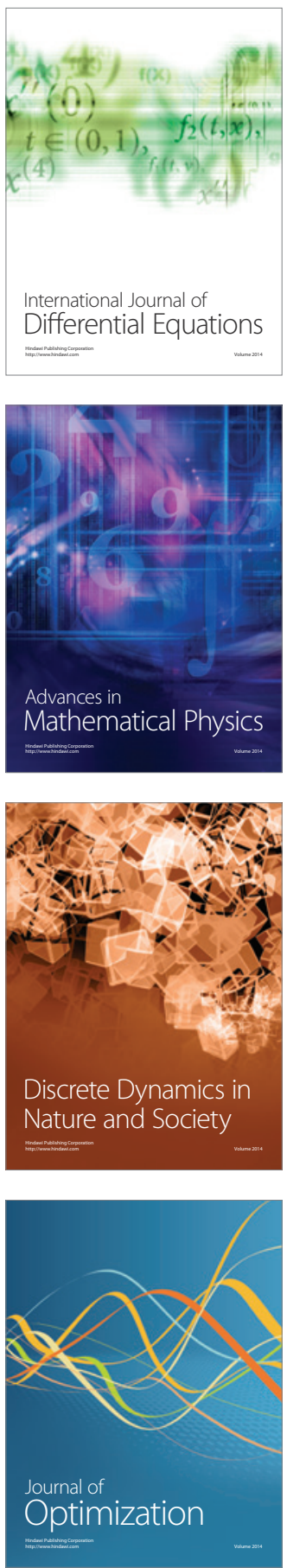